Bull. Korean Math. Soc. 39 (2002), No. 1, Pp. 63-69

\title{
TRACE-CLASS INTERPOLATION FOR VECTORS IN TRIDIAGONAL ALGEBRAS
}

\author{
Young SoO Jo AND JOO Ho Kang
}

\begin{abstract}
Given vectors $x$ and $y$ in a Hilbert space, an interpolating operator is a bounded operator $T$ such that $T x=y$. An interpolating operator for $n$ vectors satisfies the equation $T x_{i}=y_{i}$, for $i=1,2, \cdots, n$. In this article, we obtained the following : Let $x=\left(x_{2}\right)$ and $y=\left(y_{2}\right)$ be two vectors in $\mathcal{H}$ such that $x_{i} \neq 0$ for all $i=1,2, \cdots$. Then the following statements are equivalent.

(1) There exists an operator $A$ in $\operatorname{Alg} \mathcal{L}$ such that $A x=y, A$ is a trace-class operator and every $E$ in $\mathcal{L}$ reduces $A$.

(2) $\sup \left\{\frac{\left\|\sum_{k=1}^{l} \alpha_{k} E_{k} y\right\|}{\left\|\sum_{k_{k}=1}^{l} \alpha_{k} E_{k} x\right\|} ; l \in N, \alpha_{k} \in \mathbb{C}\right.$ and $\left.E_{k} \in \mathcal{L}\right\}<\infty$ and $\sum_{n=1}^{\infty}\left|y_{n}\right|\left|x_{n}\right|^{-1}<\infty$.
\end{abstract}

\section{Introduction}

Let $\mathcal{C}$ be a collection of operators acting on a Hilbert space $\mathcal{H}$ and let $x$ and $y$ be vectors on $\mathcal{H}$. An interpolation question for $\mathcal{C}$ asks for which $x$ and $y$ is there a bounded operator $T \in \mathcal{C}$ such that $T x=y$. A variation, the ' $n$-vector interpolation problem', asks for an operator $T$ such that $T x_{i}=y_{i}$ for fixed finite collections $\left\{x_{1}, x_{2}, \cdots, x_{n}\right\}$ and $\left\{y_{1}, y_{2}, \cdots, y_{n}\right\}$. The $n$-vector interpolation problem was considered for a $C^{*}$-algebra $\mathcal{U}$ by Kadison [9]. In case $\mathcal{U}$ is a nest algebra, the (onevector) interpolation problem was solved by Lance [10]: his result was extended by Hopenwasser [4] to the case that $\mathcal{U}$ is a CSL-algebra.

In this article, we investigate Trace-class interpolation problems for vectors in tridiagonal algebra: Given vectors $x$ and $y$ in a Hilbert space,

Received August 17, 2001.

2000 Mathematics Subject Classification: 47L35.

Key words and phrases: trace-class, tridiagonal algebra, commutative subspace lattice, $\operatorname{Alg} \mathcal{L}$. 
when does there exist a Trace-class operator $A$ in a tridiagonal algebra such that $A x=y$ ?

We establish some notations and conventions. A commutative subspace lattice $\mathcal{L}$, or CSL $\mathcal{L}$ is a strongly closed lattice of pairwise-commuting projections acting on a Hilbert space $\mathcal{H}$. We assume that the projections 0 and $I$ lie in $\mathcal{L}$. We usually identify projections and their ranges, so that it makes sense to speak of an operator as leaving a projection invariant. If $\mathcal{L}$ is CSL, $\operatorname{Alg} \mathcal{L}$ is called a CSL-algebra. The symbol $\operatorname{Alg} \mathcal{L}$ is the algebra of all bounded linear operators on $\mathcal{H}$ that leave invariant all the projections in $\mathcal{L}$. Let $x$ and $y$ be two vectors in some Hilbert space. Then $\langle x, y\rangle$ means the inner product of the vectors $x$ and $y$. Let $N$ be the set of all natural numbers and let $\mathbb{C}$ be the set of all complex numbers.

\section{Trace-class interpolation for vectors in tridiagonal algebra}

Let $\mathcal{H}$ be a separable complex Hilbert space with a fixed orthonormal basis $\left\{e_{1}, e_{2}, \cdots\right\}$. Let $x_{1}, x_{2}, \cdots, x_{n}$ be vectors in $\mathcal{H}$. Then $\left[x_{1}, x_{2}\right.$, $\left.\cdots, x_{n}\right]$ means the closed subspace generated by the vectors $x_{1}, x_{2}, \cdots$, $x_{n}$. Let $M$ be a subset of a Hilbert space $\mathcal{H}$. Then $\bar{M}$ means the closure of $M$ and $\bar{M}^{\perp}$ means the orthogonal complement of $M$. Let $\mathcal{L}$ be the subspace lattice of orthogonal projections generated by the subspaces $\left[e_{2 k-1}\right],\left[e_{2 k-1}, e_{2 k}, e_{2 k+1}\right](k=1,2, \cdots)$. Then the algebra $\operatorname{Alg} \mathcal{L}$ is called a tridiagonal algebra which was introduced by F. Gilfeather and D. Larson [3]. These algebras have been found to be useful counterexample to a number of plausible conjectures. Recently, such algebras have been found to be use in physics, in electrical engineering and in general system theory.

Let $\mathcal{A}$ be the algebra consisting of all bounded operators acting on $\mathcal{H}$ of the form

$$
\left(\begin{array}{ccccc}
* & * & & & \\
& * & & & \\
& * & * & * & \\
& & * & \\
& & * & \ddots
\end{array}\right)
$$

with respect to the orthonormal basis $\left\{e_{1}, e_{2}, \cdots\right\}$, where all non-starred entries are zero. It is easy to see that $\operatorname{Alg} \mathcal{L}=\mathcal{A}$. Let

$$
D=\{A: A \text { is a diagonal operator in } \mathcal{B}(\mathcal{H})\}
$$


Then $D$ is a masa (maximal abelian subalgebra) of $\operatorname{Agl} \mathcal{L}$ and $\mathcal{D}=(\operatorname{Alg} \mathcal{L}) \cap$ $(\operatorname{Alg} \mathcal{L})^{*}$, where $(\operatorname{Alg} \mathcal{L})^{*}=\left\{A^{*}: A \in \operatorname{Alg} \mathcal{L}\right\}$.

In this paper, we use the convention $\frac{0}{0}=0$, when necessary.

Definition. Let $\mathcal{H}$ be a Hilbert space and let $A$ be an operator acting on $\mathcal{H}$. $A$ is called positive if $\langle A x, x\rangle \geq 0$ for all $x$ in $\mathcal{H}$.

DEFINITION. Let $\mathcal{H}$ be a Hilbert space, $\left\{e_{n}\right\}_{n=1}^{\infty}$ an orthonormal basis. Then for any positive operator $A$ acting on $\mathcal{H}$, we define $\operatorname{tr} A=$ $\sum_{n=1}^{\infty}\left\langle e_{n}, A e_{n}\right\rangle$. The number $\operatorname{tr} A$ is called the trace of $A$.

Definition. Let $\mathcal{H}$ be a Hilbert space and let $A$ be an operator acting on $\mathcal{H}$. $A$ is called a Hilbert-Schmidt operator if $\operatorname{tr} A^{*} A<\infty$.

Definition. Let $\mathcal{H}$ be a Hilbert space and let $\mathcal{B}(\mathcal{H})$ be the set of all bounded operators acting on $\mathcal{H}$. Let $\mathcal{B}_{2}(\mathcal{H})$ be the set of all HilbertSchmidt operators acting on $\mathcal{H}$. Let $\mathcal{B}_{1}(\mathcal{H})=\left\{A B \mid A, B \in \mathcal{B}_{2}(\mathcal{H})\right\}$. Operators belonging to $\mathcal{B}_{1}(\mathcal{H})$ are called trace-class operators.

The following theorem is well-known.

THEOREM 1. Let $\mathcal{H}$ be a Hilbert space and let $A$ be an operator in $\mathcal{B}(\mathcal{H})$. Then the following are equivalent.

(1) $A \in \mathcal{B}_{1}(\mathcal{H})$.

(2) $|A|=\left(A^{*} A\right)^{\frac{1}{2}} \in \mathcal{B}_{1}(\mathcal{H})$.

(3) $|A|^{\frac{1}{2}} \in \mathcal{B}_{2}(\mathcal{H})$

(4) $\operatorname{tr}(|A|)<\infty$.

From Theorem 1, we can get the following theorem.

THEOREM 2. Let $A$ be a diagonal operator in $\mathcal{B}(\mathcal{H})$ with diagonal $\left\{a_{n}\right\}$. $A$ is a trace-class operator if and only if $\sum_{n=1}^{\infty}\left|a_{n}\right|<\infty$.

TheOREM 3. Let $x=\left(x_{i}\right)$ and $y=\left(y_{i}\right)$ be two vectors in $\mathcal{H}$ such that $x_{i} \neq 0$ for all $i=1,2, \cdots$. If

$$
\sup \left\{\frac{\left\|\sum_{k=1}^{l} \alpha_{k} E_{k} y\right\|}{\left\|\sum_{k=1}^{l} \alpha_{k} E_{k} x\right\|}: l \in N, \alpha_{k} \in \mathbb{C} \text { and } E_{k} \in \mathcal{L}\right\}<\infty
$$

and

$$
\sum_{n=1}^{\infty}\left|y_{n}\right|\left|x_{n}\right|^{-1}<\infty
$$


then there is an operator $A$ in $A \lg \mathcal{L}$ such that $A x=y$, every $E$ in $\mathcal{L}$ reduces $A$ and $A$ is a trace-class operator.

Proof. If $\sup \left\{\frac{\left\|\sum_{k=1}^{l} \alpha_{k} E_{k} y\right\|}{\left\|\sum_{k=1}^{l} \alpha_{k} E_{k} x\right\|}: l \in N, \alpha_{k} \in \mathbb{C}\right.$ and $\left.E_{k} \in \mathcal{L}\right\}<\infty$, then, there is an operator $A$ in $\operatorname{Alg} \mathcal{L}$ such that $A x=y$ and every $E$ in $\mathcal{L}$ reduces $A$ by Theorem 1 [8]. Since every $E$ in $\mathcal{L}$ reduces $A, A$ is diagonal. Let $A=\left(a_{i i}\right)$. Since $y=A x, y_{i}=a_{i i} x_{i}$ for all $i=1,2, \cdots$. Since $\sum_{n=1}^{\infty}\left|y_{n}\right|\left|x_{n}\right|^{-1}<\infty, A$ is a trace-class operator.

THEOREM 4. Let $x=\left(x_{i}\right)$ and $y=\left(y_{i}\right)$ be two vectors in $\mathcal{H}$ such that $x_{i} \neq 0$ for all $i=1,2, \cdots$. If there exists an operator $A$ in $A \lg \mathcal{L}$ such that $A x=y$, every $E$ in $\mathcal{L}$ reduces $A$ and $A$ is a trace-class operator, then

$$
\sup \left\{\frac{\left\|\sum_{k=1}^{l} \alpha_{k} E_{k} y\right\|}{\left\|\sum_{k=1}^{l} \alpha_{k} E_{k} x\right\|} ; l \in N, \alpha_{k} \in \mathbb{C} \text { and } E_{k} \in \mathcal{L}\right\}<\infty
$$

and

$$
\sum_{n=1}^{\infty}\left|y_{n}\right|\left|x_{n}\right|^{-1}<\infty
$$

Proof. Since $A x=y$ and every $E$ in $\mathcal{L}$ reduces $A, A E x=E y$ for every $E$ in $\mathcal{L}$. So $A\left(\sum_{k=1}^{l} \alpha_{k} E_{k} x\right)=\sum_{k=1}^{l} \alpha_{k} E_{k} y$ for every $l \in N$, every $\alpha_{k} \in \mathbb{C}$ and every $E_{k} \in \mathcal{L}$. Thus $\left\|\sum_{k=1}^{l} \alpha_{k} E_{k} y\right\| \leq\|A\|\left\|\sum_{k=1}^{l} \alpha_{k} E_{k} x\right\|$. If $\left\|\sum_{k=1}^{l} \alpha_{k} E_{k} x\right\| \neq 0$, then

$$
\frac{\left\|\sum_{k=1}^{l} \alpha_{k} E_{k} y\right\|}{\left\|\sum_{k=1}^{l} \alpha_{k} E_{k} x\right\|} \leq\|A\| .
$$

Hence

$$
\sup \left\{\frac{\left\|\sum_{k=1}^{l} \alpha_{k} E_{k} y\right\|}{\left\|\sum_{k=1}^{l} \alpha_{k} E_{k} x\right\|}: l \in N, \alpha_{k} \in \mathbb{C} \text { and } E_{k} \in \mathcal{L}\right\}<\infty .
$$

Since every $E$ in $\mathcal{L}$ reduces $A, A$ is diagonal. Let $A=\left(a_{i i}\right)$. Since $A x=y, y_{i}=a_{i i} x_{i}$ and hence $a_{i i}=y_{i} x_{i}^{-1}$ for all $i=1,2, \cdots$. Since $A$ is a trace-class operator, $\sum_{n=1}^{\infty}\left|y_{n}\right|\left|x_{n}\right|^{-1}<\infty$.

If we summarize Theorems 3 and 4 , then we can get the following theorem. 
THEOREM 5. Let $x=\left(x_{i}\right)$ and $y=\left(y_{i}\right)$ be two vectors in $\mathcal{H}$ such that $x_{i} \neq 0$ for all $i=1,2, \cdots$. Then the following statements are equivalent.

(1) There exists an operator $A$ in $A \lg \mathcal{L}$ such that $A x=y, A$ is a trace-class operator and every $E$ in $\mathcal{L}$ reduces $A$.

(2) $\sup \left\{\frac{\left\|\sum_{k=1}^{l} \alpha_{k} E_{k} y\right\|}{\left\|\sum_{k=1}^{l} \alpha_{k} E_{k} x\right\|}: l \in N, \alpha_{k} \in \mathbb{C}\right.$ and $\left.E_{k} \in \mathcal{L}\right\}<\infty$ and $\sum_{n=1}^{\infty}\left|y_{n}\right|\left|x_{n}\right|^{-1}<\infty$

THEOREM 6. Let $x_{p}=\left(x_{p, i}\right)$ and $y_{p}=\left(y_{p, i}\right)$ be vectors in $\mathcal{H}$ such that $x_{q, i} \neq 0(p=1,2, \cdots, n)$ for some fixed $q$ and all $i=1,2, \cdots$. If there is an operator $A$ in $A \lg \mathcal{L}$ such that $A x_{p}=y_{p}(p=1,2, \cdots, n)$, every $E$ in $\mathcal{L}$ reduces $A$ and $A$ is a trace-class operator, then $\sup \left\{\frac{\left\|\sum_{k=1}^{m_{p}} \sum_{p=1}^{l} \alpha_{k, p} E_{k, p} y_{p}\right\|}{\left\|\sum_{k=1}^{m_{p}} \sum_{p=1}^{l} \alpha_{k, p} E_{k, p} x_{p}\right\|}: m_{p} \in N, l \leq n, E_{k, p} \in \mathcal{L}\right.$ and $\left.\alpha_{k, p} \in \mathbb{C}\right\}$ $<\infty$ and $\sum_{n=1}^{\infty}\left|y_{q, n}\right|\left|x_{q, n}\right|^{-1}<\infty$.

Proof. Since $A x_{p}=y_{p}$ and every $E$ in $\mathcal{L}$ reduces $A, A E x_{p}=E y_{p}$. So $A\left(\sum_{k=1}^{m_{p}} \sum_{p=1}^{l} \alpha_{k, p} E_{k, p} x_{p}\right)=\sum_{k=1}^{m_{p}} \sum_{p=1}^{l} \alpha_{k, p} E_{k, p} y_{p}, m_{p} \in N, l \leq$ $n, E_{k, p} \in \mathcal{L}$ and $\alpha_{k, p} \in \mathbb{C}$. Thus $\left\|\sum_{k=1}^{m_{p}} \sum_{p=1}^{l} \alpha_{k, p} E_{k, p} y_{p}\right\| \leq \| \sum_{k=1}^{m_{p}}$ $\sum_{p=1}^{l} \alpha_{k, p} E_{k, p} x_{p}\|\| A \|$. If $\left\|\sum_{k=1}^{m_{p}} \sum_{p=1}^{l} \alpha_{k, p} E_{k, p} x_{p}\right\| \neq 0$, then

$$
\frac{\left\|\sum_{k=1}^{m_{p}} \sum_{p=1}^{l} \alpha_{k, p} E_{k, p} y_{p}\right\|}{\left\|\sum_{k=1}^{m_{p}} \sum_{p=1}^{l} \alpha_{k, p} E_{k, p} x_{p}\right\|} \leq\|A\|
$$

Hence

$\sup \left\{\frac{\left\|\sum_{k=1}^{m_{p}} \sum_{p=1}^{l} \alpha_{k, p} E_{k, p} y_{p}\right\|}{\left\|\sum_{k=1}^{m_{p}} \sum_{p=1}^{l} \alpha_{k, p} E_{k, p} x_{p}\right\|}: m_{p} \in N, l \leq n, E_{k, p} \in \mathcal{L}\right.$ and $\left.\alpha_{k, p} \in \mathbb{C}\right\}$ $<\infty$. Since every $E$ in $\mathcal{L}$ reduces $A, A$ is diagonal. Let $A=\left(a_{i i}\right)$. Since $A x_{p}=y_{p}, y_{p, i}=a_{i i} x_{p, i}(p=1,2, \cdots, n$ and $i=1,2, \cdots)$. Since $x_{q, i} \neq 0, a_{i i}=y_{q, i} x_{q, i}^{-1}(i=1,2, \cdots)$. Since $A$ is a trace-class operator, $\sum_{n=1}^{\infty}\left|y_{q, n}\right|\left|x_{q, n}\right|^{-1}<\infty$

THEOREM 7. Let $x_{p}=\left(x_{p, i}\right)$ and $y_{p}=\left(y_{p, i}\right)$ be vectors in $\mathcal{H}$ such that $x_{q, i} \neq 0(p=1,2, \cdots, n)$ for some fixed $q$ and all $i=1,2, \cdots$. 
If $\sup \left\{\frac{\left\|\sum_{k=1}^{m_{p}} \sum_{p=1}^{l} \alpha_{k, p} E_{k, p} y_{p}\right\|}{\left\|\sum_{k=1}^{m_{p}} \sum_{p=1}^{l} \alpha_{k, p} E_{k, p} x_{p}\right\|}: m_{p} \in N, l \leq n, E_{k, p} \in \mathcal{L}\right.$ and $\left.\alpha_{k, p} \in \mathbb{C}\right\}<\infty$ and $\sum_{n=1}^{\infty}\left|y_{q, n}\right|\left|x_{q, n}\right|^{-1}<\infty$, then there is an operator $A$ in $A \lg \mathcal{L}$ such that $A x_{p}=y_{p}(p=1,2, \cdots, n)$, every $E$ in $\mathcal{L}$ reduces $A$ and $A$ is a trace-class operator.

Proof. If $\sup \left\{\frac{\left\|\sum_{k=1}^{m_{p}} \sum_{p=1}^{l} \alpha_{k, p} E_{k, p} y_{p}\right\|}{\left\|\sum_{k=1}^{m_{p}} \sum_{p=1}^{l} \alpha_{k, p} E_{k, p} x_{p}\right\|}: m_{p} \in N, l \leq n, E_{k, p} \in \mathcal{L}\right.$ and $\left.\alpha_{k, p} \in \mathbb{C}\right\}<\infty$, then there is an operator $A$ in $\operatorname{Alg} \mathcal{L}$ such that $A x=y$ and every $E$ in $\mathcal{L}$ reduces $A$. So $A$ is a diagonal operator. Let $A=\left(a_{i i}\right)$. Since $y_{p}=A x_{p}, y_{p, i}=a_{i i} x_{p, i}(p=1,2, \cdots, n$ and $i=$ $1,2, \cdots)$. Since $\sum_{n=1}^{\infty}\left|y_{q, n}\right|\left|x_{q, n}\right|^{-1}<\infty, A$ is a trace-class operator.

If we summarize Theorems 6 and 7 , then we can get the following theorem.

THEOREM 8. Let $x_{p}=\left(x_{p, i}\right)$ and $y_{p}=\left(y_{p, i}\right)$ be vectors in $\mathcal{H}$ such that $x_{q, i} \neq 0$ for some fixed $q$ and all $i=1,2, \cdots$. Then the following statements are equivalent.

(1) There exists an operator $A$ in Alg $\mathcal{L}$ such that $A x_{p}=y_{p}(p=$ $1, \cdots, n)$, every $E$ in $\mathcal{L}$ reduces $A$ and $A$ is a trace-class operator.

(2) $\sup \left\{\frac{\left\|\sum_{k=1}^{m_{p}} \sum_{p=1}^{l} \alpha_{k, p} E_{k, p} y_{p}\right\|}{\left\|\sum_{k=1}^{m_{p}} \sum_{p=1}^{l} \alpha_{k, p} E_{k, p} x_{p}\right\|}: m_{p} \in N, l \leq n, E_{k, p} \in \mathcal{L}\right.$ and $\left.\alpha_{k, p} \in \mathbb{C}\right\}<\infty$ and $\sum_{n=1}^{\infty}\left|y_{q, n}\right|\left|x_{q, n}\right|^{-1}<\infty$.

If we modify the proof of Theorems 6 and 7 , then we can get the following theorem that is considered for infinite vectors.

THEOREM 9. Let $x_{p}=\left(x_{p, i}\right)$ and $y_{p}=\left(y_{p, i}\right)$ be vectors in $\mathcal{H}(p=$ $1,2, \cdots)$ such that $x_{q, i} \neq 0$ for all $i$ and for some fixed $q$. Then the following statements are equivalent.

(1) There exists an operator $A$ in Alg $\mathcal{L}$ such that $A x_{p}=y_{p}(p=$ $1,2, \cdots)$, every $E$ in $\mathcal{L}$ reduces $A$ and $A$ is a trace-class operator. 
(2) $\sup \left\{\frac{\left\|\sum_{k=1}^{m_{p}} \sum_{p=1}^{l} \alpha_{k, p} E_{k, p} y_{p}\right\|}{\left\|\sum_{k=1}^{m_{p}} \sum_{p=1}^{l} \alpha_{k, p} E_{k, p} x_{p}\right\|}: m_{p}, l \in N, E_{k, p} \in \mathcal{L}\right.$ and $\alpha_{k, p} \in$ $\mathbb{C}\}<\infty$ and $\sum_{n=1}^{\infty}\left|y_{q, n}\right|\left|x_{q, n}\right|^{-1}<\infty$.

\section{References}

[1] W. B. Arveson, Interpolation problems in nest algebras, J. Functional Analysis 3 (1975), 208-233.

[2] R. G. Douglas, On majorization, factorization, and range inclusion of operators on Hilbert space, Proc. Amer. Math. Soc. 17 (1966), 413-415.

[3] F. Gilfeather and D. Larson, Commutants modulo the compact operators of certain CSL algebras, Operator Theory: Adv. Appl. 2 (Birkhauser, Basel, 1981), 105-120.

[4] A. Hopenwasser, The equation $T x=y$ in a reflexive operator algebra, Indiana University Math. J, 29 (1980), 121-126.

[5] __ Hilbert-Schmidt interpolation in CSL algebras, Illinois J. Math. $\mathbf{3 3}$ (1989), no. 4, 657-672.

[6] Y.S. Jo, Isometries of Tridiagonal Algebras, Pacific Journal of Mathematics 140 (1989), no. 1, 97-115.

[7] Y.S. Jo and T. Y. Choi, Isomorphisms of $A \lg \mathcal{L}_{n}$ and $A l g \mathcal{L}_{\infty}$, Michigan Math. J. 37 (1990), 305-314.

[8] Y.S. Jo and J. H. Kang, Interpolation problems in CSL-Algebra AlgL, to appear in Rocky Mountain Journal of Math.

[9] R. Kadison, Irreducible Operator Algebras, Proc. Nat. Acad. Sci. U.S.A. (1957), 273-276.

[10] E. C. Lance, Some properties of nest algebras, Proc. London Math. Soc., 3, 19 (1969), 45-68.

Young Soo Jo, Department of Mathematics, Keimyung University, Taegu 704"701, KOREA

E-mail: ysjo@kmu.ac.kr

Joo Ho Kang, Department of Mathematics, TaEgu University, Taggu 712 -

714, KOREA

E-mail: jhkang@taegu.ac.kr 